\section{Nucleoid organization}

Prokaryotes do not compartmentalize their genomic DNA within a membrane-enclosed nucleus, but that doesn't mean that their genomes are not organized. In fact, bacterial DNA occupies only the central part of the cell, forming the socalled nucleoid. In addition,

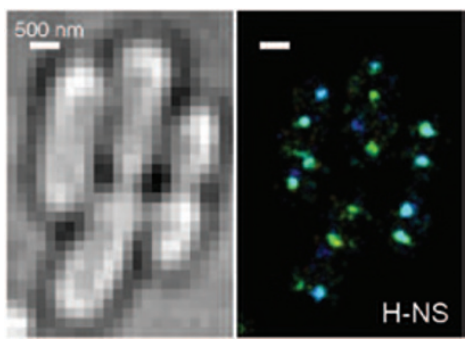
some loci have been observed in specific positions, especially during DNA replication and segregation. However, bacterial cells are quite small, and a detailed characterization of the nucleoid architecture has been hampered by technical limitations. Now Zhuang, Xie and colleagues have used super-resolution fluorescence microscopy to study the distribution of five nucleoid-associated proteins from Escherichia coli: HU, Fis, IHF, StpA and H-NS. Each of these proteins was tagged with the monomeric photoactivatable fluorescent protein mEos 2 and expressed in E. coli. The first four proteins showed a scattered distribution within the nucleoid. In contrast, H-NS was concentrated in two clusters per nucleoid, localized near the one-quarter and three-quarter positions along the long axis of the newly-divided, rod-shaped cells. H-NS has an $\mathrm{N}$-terminal domain that mediates homo-oligomerization, and the authors showed by mutagenesis that this is necessary for cluster formation. $\mathrm{H}-\mathrm{NS}$ is involved in transcriptional silencing. To gain insight into how cluster organization and transcriptional control by H-NS are related, the authors followed the positions of two genes regulated by H-NS by inserting tet operator sequences upstream of the genes and expressing a fluorescent protein fused to the Tet repressor. They found that these two gene loci tended to localize with the H-NS clusters, even though they were distant from each other along the chromosome, and this localization required $\mathrm{H}$-NS. However, the loci were also quite mobile and did not always localize with the H-NS cluster, indicating their dynamic nature. The pairwise proximity of several H-NS-regulated genes was independently established using chromosome conformation capture (3C) assays. Exploring the organization of the bacterial genome at such a resolution is a technical feat, and this work will lead to a better understanding of genomic architecture and regulation in bacteria. (Science 333, 1445-1449, 2011)

IC

\section{Sense after shifting}

Cells alter their behavior in response to physiological needs or when they detect exogenous small molecules (such as essential nutrients or deleterious toxins) through a broad range of mechanisms, including those of transmembrane receptors or feedback inhibition of enzymes in metabolic pathways. Polyamines are small organic compounds that possess multiple positive charges at physiological $\mathrm{pH}$ levels and help regulate cellular proliferation and differentiation. The first step in polyamine biosynthesis involves ornithine decarboxylase (ODC), an enzyme that converts ornithine to putrescine. ODC is regulated by the ODC antizyme, a small protein that targets ODC for ubiquitin-independent degradation by the proteasome. High intracellular concentrations of polyamines lead to an increase in the production of the ODC antizyme, which inhibits the activity of ODC and, thus, polyamine biosynthesis. Dohmen and colleagues now report how polyamines regulate the translation of the mRNA for OAZ1, the sole ODC antizyme in Saccharomyces cerevisiae. Translation of the full-length OAZ1 mRNA only occurs if a ribosomal frameshift event takes place in the middle of the transcript. The authors found that changes to

Written by Inês Chen, Joshua M Finkelstein \& Arianne Heinrichs the $5^{\prime}$ portion of the OAZ1 mRNA altered the efficiency of ribosomal frameshifting, but only the $3^{\prime}$ region of the transcript 'sensed' the presence of polyamines. Silent mutations in the OAZ1 mRNA 3' portion did not alter polyamine-sensing, but mutations that changed residues very close to C-terminus of the OAZ1 polypeptide did. These observations revealed that polyamines directly bind to the nascent polypeptide while still associated with the ribosome-mRNA complex. The authors propose that when polyamine concentrations are low, depending on translational pausing caused by the frameshifting site, the nascent OAZ1 polypeptide assumes a conformation that leads to translational arrest; higher polyamine concentrations somehow prevent formation of this inhibitory conformation, allowing translation of full-length ODC antizyme to transpire. The exact mechanism by which this occurs is not yet clear, nor is it clear whether this unusual mRNA translational mechanism is unique to polyamines or whether other metabolites use this process to regulate enzymes in their own biosynthetic pathways. (Nature doi:10.1038/ nature10393, published online 7 September 2011)

$J M F$

\section{Signaling through chromatin}

Trimethylation of histone $\mathrm{H} 3 \mathrm{~K} 4$, which marks active, transcribed genes, is catalyzed by the methyltransferase complex COMPASS and regulated in trans by prior ubiquitination of histone H2B Lys123 (H2BK123). The kinetochore protein Dam1, a component of the Dam1 (or DASH) complex that anchors kinetochores to microtubules, is the only other known substrate of COMPASS. Latham et al. now show that Dam1 dimethylation on K233 requires intact COMPASS and prior ubiquitination of $\mathrm{H} 2 \mathrm{BK} 123$ in budding yeast, suggesting that histone modifications can direct modifications in non-histone proteins. Indeed, genetic deletion of the Rad6 E2 ubiquitin-conjugating enzyme responsible for $\mathrm{H} 2 \mathrm{BK} 123$ ubiquitination or its corresponding E3 ligase Bre1 cause a phenotype associated with defective Dam 1 methylation. By contrast, genetic deletion of the ubiquitin protease Ubp8 results in increased Dam1 dimethylation levels. The Paf 1 transcription elongation complex, which is required for recruiting COMPASS and subsequent di- and trimethylation of $\mathrm{H} 3 \mathrm{~K} 4$ at gene promoters, is also required for Dam 1 methylation. This raises the question of whether transcription plays a role in Dam 1 methylation. Whereas $\mathrm{H} 2 \mathrm{BK} 123$ ubiquitination and COMPASS recruitment to specific gene promoters require active RNA polymerase II (Pol II) transcription and the activity of Kin28 kinase (which phosphorylates the C-terminal domain of Pol II, marking the transition from transcription initiation to elongation), Dam 1 methylation is independent of Kin28 activity. This suggests that Paf 1 has functions independent of transcription in regulating kinetochore function, which leads to the question of how H3BK123 ubiquitination activity and the Paf complex are targeted to the centromere. It is possible that another kinase is required or that recruitment occurs through a Pol II-independent mechanism. Also, methylation of Dam 1 occurs only in a functional kinetochore context, as mutations that disconnect the kinetochore from the centromere or the Dam 1 complex from the rest of the kinetochore affect Dam 1 methylation. The authors speculate that Dam 1 methylation might be part of a phospho-methyl switch regulating kinetochore function and chromosome segregation, whereby methylation of Dam 1 inhibits phosphorylation of flanking serine residues by Ipl1 kinase. (Ce// 146, 709-719, 2011) 